\title{
Real-time Display System to Monitor Plasma Shape and Position During the Discharge of the Tokamak
}

\author{
Md Mahbub Alam ${ }^{1, ~ *, ~ M d ~ D u l a l ~ H o s s a i n ~}{ }^{1}$, Md Shafiul Alam Chowdhury ${ }^{2}$ \\ ${ }^{1}$ Institute of Computer Science, Bangladesh Atomic Energy Commission, Agargaon, Dhaka, Bangladesh \\ ${ }^{2}$ Department of Computer Science and Engineering, Uttara University, Uttara, Dhaka, Bangladesh
}

Email address:

mahbub.baec@gmail.com(Md M. Alam), edhossain@gmail.com(Md D. Hossain), shafiul.a.chowdhury@gmail.com(Md S. A. Chowdhury)

${ }^{*}$ Corresponding author

To cite this article:

Md Mahbub Alam, Md Dulal Hossain, Md Shafiul Alam Chowdhury. Real-time Display System to Monitor Plasma Shape and Position During the Discharge of the Tokamak. International Journal of Engineering Management. Vol. 3, No. 1, 2019, pp. 12-16.

doi: $10.11648 /$ j.ijem.20190301.13

Received: June 6, 2019; Accepted: July 10, 2019; Published: July 24, 2019

\begin{abstract}
A novel, efficient and precise real-time display system to monitor the plasma shape and position during the discharge of the tokamak has been offered in this study for the observation of the continuous behavior of the plasma that is produced inside the vacuum vessel. The observed behavior of the plasma can provide indications for the control and operation of the tokamak to achieve long time discharge. The display system can display the plasma cross-sectional view of the last closed flux surface (LCFS) with the position of the vacuum vessel wall and the X-points position with the setting of the divertor plates at a big screen in real-time. The display system offers not only the visual image of the plasma but also time evolution graphs of various plasma parameters such as the plasma current $\left(I_{P}\right)$, poloidal field coils currents $\left(I_{P F}\right)$, emission of $H_{\alpha}$, transport of the oxygen impurity, major radius $(R)$, minor radius $(a)$, plasma elongation $(\kappa)$ and triangularity $(\delta)$ in real-time. For the remote participation to the experiments, the display system has a subsystem that can record the entire display frame and the time evolution graphs of the various plasma parameters as video files. The recorded video files are accessible through online by the remote participants during the operation of the tokamak. In addition, the display system has an emergency safety notification system that can identify the critical condition of the tokamak during its operation and can notify the occurrences of any critical or abnormal situations by generating an alarm. The overall display system and its subsystems have been designed and integrated with the real-time hardware equipment of the National Instruments Corporation (NI) and the entire data acquisition and computational systems have been developed by the LabVIEW programming Language.
\end{abstract}

Keywords: Display System, Long Time Discharge, Plasma Shape, Position, Real-time, Tokamak, Safety Notification System

\section{Introduction}

The identification of the plasma shape and the position in real-time is important during the operation of the tokamak to achieve long time discharge. By observing the temporal behaviors of the plasma shape and position in real-time, the experimenters can get indications about what measures should be taken into account to achieve steady state operation as well as long time discharge [1]. Usually high speed cameras have been used to observe the plasma shape and position during the operation of the tokamak, but it is difficult to identify the magnetic surface and its position from the cameras' images accurately. Whereas the real-time display system offered plasma cross-sectional view during the operation of the tokamak in a big screen placed at the control room of the tokamak in real-time. The experimenters can get indications about the difference between the plasma and the vessel wall, the X-points position and connection of the magnetic line to the divertor plates [2].

In the International Thermonuclear Experimental Reactor (ITER), a Real-Time Plasma Boundary Display System (RBDS) is going to develop around the year 2030 to display graphs, figures, colors and a cutaway view of the vacuum vessel as a development of ITER control room [3]. In the Experimental Advanced Superconducting Tokamak (EAST), 
data acquisition and remote real-time display system for EAST NBI has been developed for its safe and smooth operation. Later a real-time image acquisition system has been developed at EAST to obtain plasma radiation image during the discharge processes. This image represents the plasma position and boundary with high accuracy, which is essential to the success of Tokamak discharge $[4,5]$. In the Joint European Torus (JET), a real time plasma boundary determination and display system using transputers has been utilized to get the valuable feedback for the operation of JET [6]. In the Wendelstein 7-X (W7-X) fusion experiment, a real-time image diagnostic system has been developed to monitor the plasma-facing components (PFCs) that can permanently damage the tokamak plasma operations. This system also has an alarm system to interlock the system [7]. In the Q-shu University experiment with steady-state spherical tokamak (QUEST), plasma control system (PCS) that is used to control and operation of the tokamak experiments has been upgraded to achieving long duration plasma sustainment [8].

\section{System Design and Plan}

For the construction of an effective real-time display system, precise and accurate calculation of the plasma shape and position from the magnetic measurements data should be ensured. The magnetic signals of the flux loops, magnetic probes and rogowski coils usually obtained as numerical integration of raw signals which are affected a critical drift error. This drift error should be removed for the precise calculation of the plasma shape and position [9]. The field-programmable gate array (FPGA) based reconfigurable real-time data acquisition module can be used to acquire flux loops, magnetic probes and rogowski coils signals through numerical integration of the raw signals by removing the drift error. FPGA is an integrated circuit that can be configured by the user. The main advantage of the FPGA modules are the high computational speed for the analog inputs and outputs signals [10]. A program written by using the algorithm of numerical integration is installed on the FPGA module that calculate magnetic fluxes of the loop voltage signals with a sampling frequency of $100 \mathrm{kHz}$. Another offset trimming routine is also installed on the FPGA module that subtracts the time-averaged offset voltage before starting of the poloidal field coil currents from the raw voltage during the numerical integration. The overall processes are shown in figure 1. As a result, the system will obtain precise and accurate magnetic measurements signals for the calculation of the plasma shape and position in real-time.

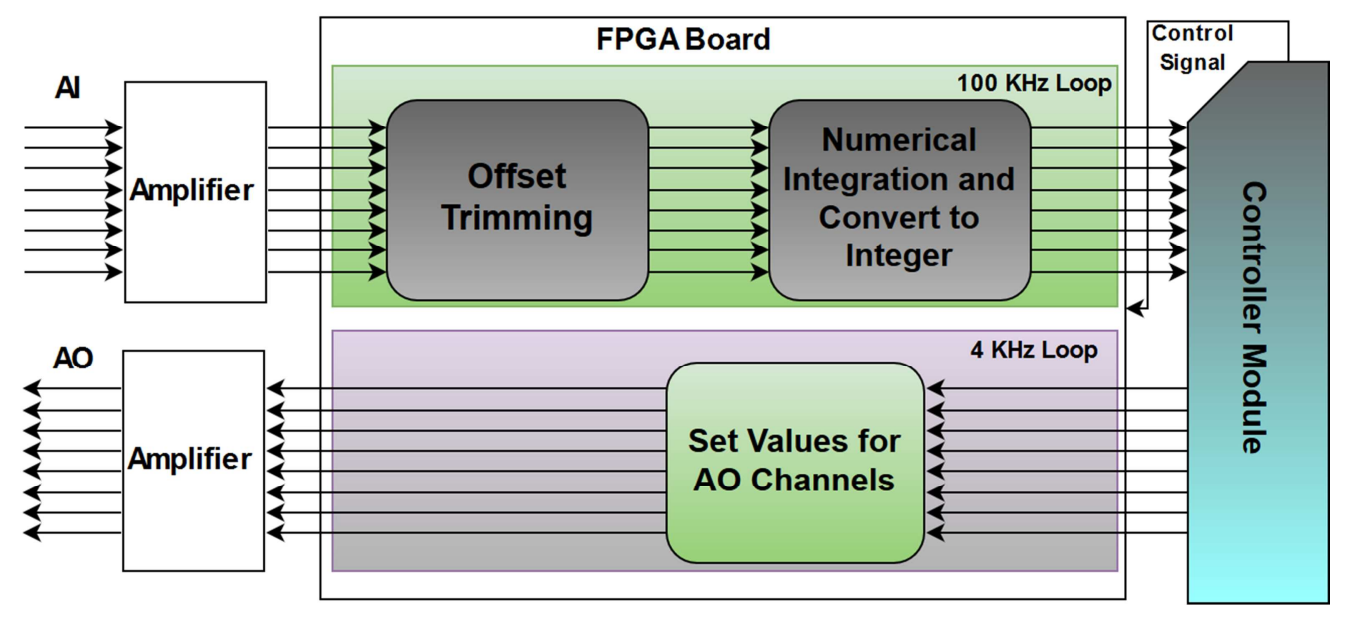

Figure 1. Functional Block Diagram of a FPGA Board.

To increase the functionality of the real-time display system, the system has two sub-systems (i) recording system (RS) and (ii) emergency safety notification system (ESNS). The RS will record the display frame of a shot as video file and store the recorded video file in the web-server. The recorded video file will be available on the online just after the shot. This function of the display system offers the researchers for the remote participation to the experiments. By using this facility, the collaborative researchers of the tokamak can observe experimental outcomes from remote places and can provide their valuable feedbacks for the operation and control of the tokamak. In addition, the RS will also store the visual image of the plasma in a database server. The researchers can use these stored video files for details analysis to understand experimental scenarios.
The ESNS will detect the over current and voltage signals, interaction of the hot plasma with the vacuum vessel and shifting the strike point's locations from the divertor plates. If any critical condition is identified, the ESNS will generate an alarm for the attention to the experimenters about the occurrences of abnormal situations.

\section{Configuration and Layout}

The real-time display system consists of mainly a real-time data acquisition and calculation system (DACS) and a real-time plasma shape, position and graphs display system (DS). The DACS is connected to the tokamak via BNC connectors. The DS is connected to the DACS via Gigabit Ethernet of the IEEE $802.3 \mathrm{Z}$ standard. The sub-systems of the 
display system such as RS is connected with the DS via digital visual interface (DVI) cable and ESNS is integrated on the DACS. The overall system layout is shown in figure 2 .

The DACS is composed of PXI systems of the National Instruments (NI) Corporation. The DACS include a PXI-8110 embedded controller module of $2.26 \mathrm{GHz}$ Quad-Core processor, four PXI-7853 multifunction reconfigurable input/output (I/O) modules, a PXI-6508 digital I/O module. Each multifunction reconfigurable I/O module has eight analog input (AI) and eight analog output (AO) channels with a user-programmable FPGA for onboard signal processing and custom system timing and synchronization. This module has a dedicated analog-to-digital converter (ADC) per channel for independent timing and triggering. The digital I/O module has 96 bidirectional channels for digital I/O. All the modules of the systems run under real-time operating system that guarantees the complete real-time data acquisition and computational facilities [11].

The DS is the Intel Core i7-7500U Processor and MS Windows operating system based general purpose computer. It has Gigabit Ethernet port for the TCP/IP communication. The $\mathrm{RS}$ is integrated by the DVI2USB 3.0TM video grabber device of Epiphan Systems with USB connected web-server. The ESNS is a general purpose computer with alarming system that can control by the computer program.

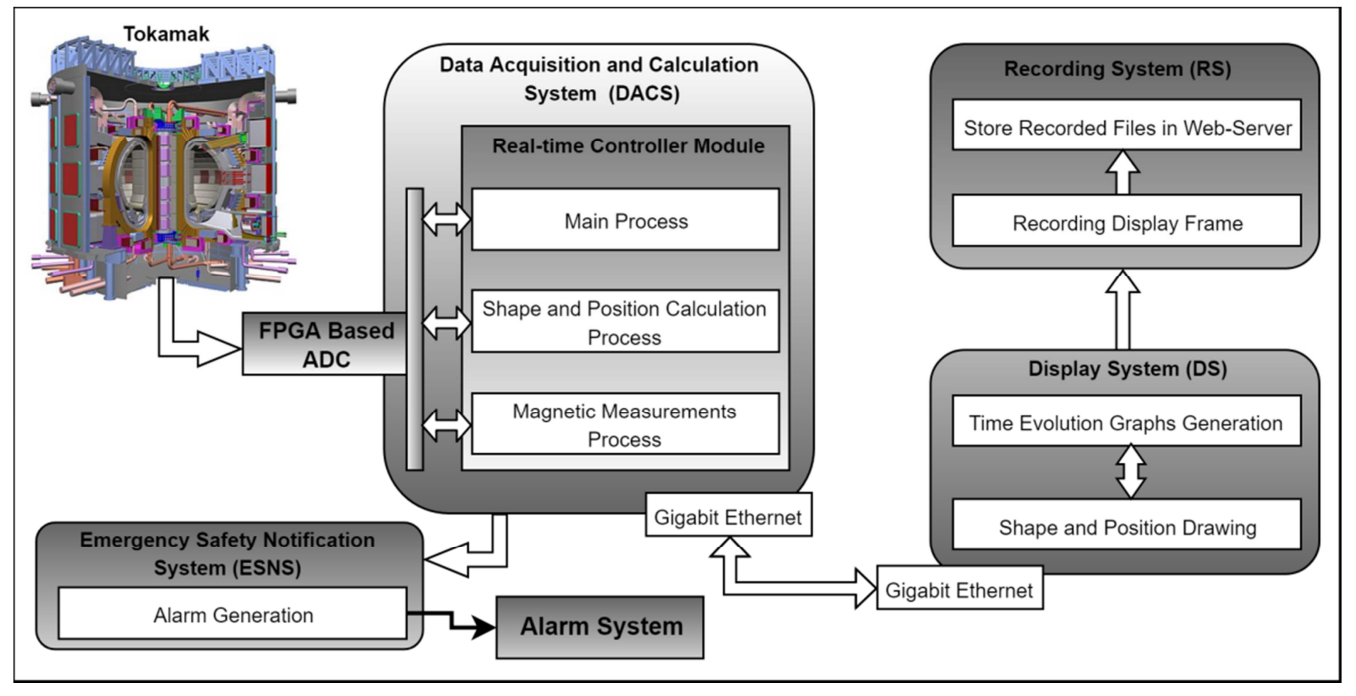

Figure 2. Block diagram of the real-time display system for the tokamak.

\section{Methods}

In the real-time DACS, several processes such as main process, shape and position calculation process and magnetic measurements process are executed. The main process also controlled the other system such as ESNS to detect the occurrence of any abnormal situation as well as to generate alarm signals. The shape and position calculation process calculated the plasma shape and position. In shape and position calculation process, two shape and position calculation methods have been used to ensure the accuracy of the real-time display system for the calculation of plasma shape and position. The magnetic measurements process controlled the data acquisition modules. On the other hand, in DS plasma shape and position drawing process and generation of time evolution graphs process are executed. The RS recorded the display frames of the display monitor as video files and stored the video files in the web-server by executing two processes in parallel.

The concepts underling in the plasma shape and position calculation and the data flow model of the real-time display system are summarized by the following points and also shown in figure 3 :

a. By the DACS, the flux loops, magnetic probes, rogowski coils data and poloidal field coils currents data are directly acquired from the tokamak.

b. Depending on the acquired data, the plasma shape basically the last closed flux surface, position and other plasma parameters calculation are performed by using the filament current method as well as the Cauchy-Condition Surface (CCS) method in parallel.

c. From the position and shape identification parameters the ESNS is calculated the occurrence of any abnormal situation. If any abnormal situation is occurred it generates an alarm signal to produce the alarm.

d. The output of the shape and position calculation parameters such as major radius, plasma position, aspect ratio, minor radius, elongation, triangularity are transferred to the DS via Gigabit Ethernet.

e. The DS draws the plasma shape especially the last closed flux surface and shows its produced position from the acquired data of the DACS. The plasma shape, position and time evolution graphs drawing are performed in the DS by the LabVIEW visual instruments programming with incorporating MATLAB programming script. The drawn figures are displayed at the big monitor connected to the DS.

f. The RS records the display frame as video files of the DVI output of DS in parallel while it is displaying at the monitor. The recorded files are stored in the web-server. 


\section{Plasma Shape and Position Calculation}

To identify the plasma shape and position, two methods are used; first, assuming the plasma current as one filament current method and second, CCS method. In QUEST, it is reported that plasma shape basically large close flux surface has been calculated by using plasma as one filament current method with fairly simple assumptions [12]. So, to identify the plasma shape and position of this real-time display system this method is used.

On the other hand, the CCS method has suitable precision for the plasma shape and position calculation in the conventional tokamak and applicable for the real-time plasma control process [13]. But for the spherical tokamak good precision can be obtained by the CCS method if we solve the inverse problem of the flux loop measurement by using least square method [14] and to remove noise we can introduce singular value decomposition on CCS method so that the CCS method can reproduce plasma boundary shape precisely with $0.5 \mathrm{~ms}$ for the real-time system by using the data from magnetic sensors [15].

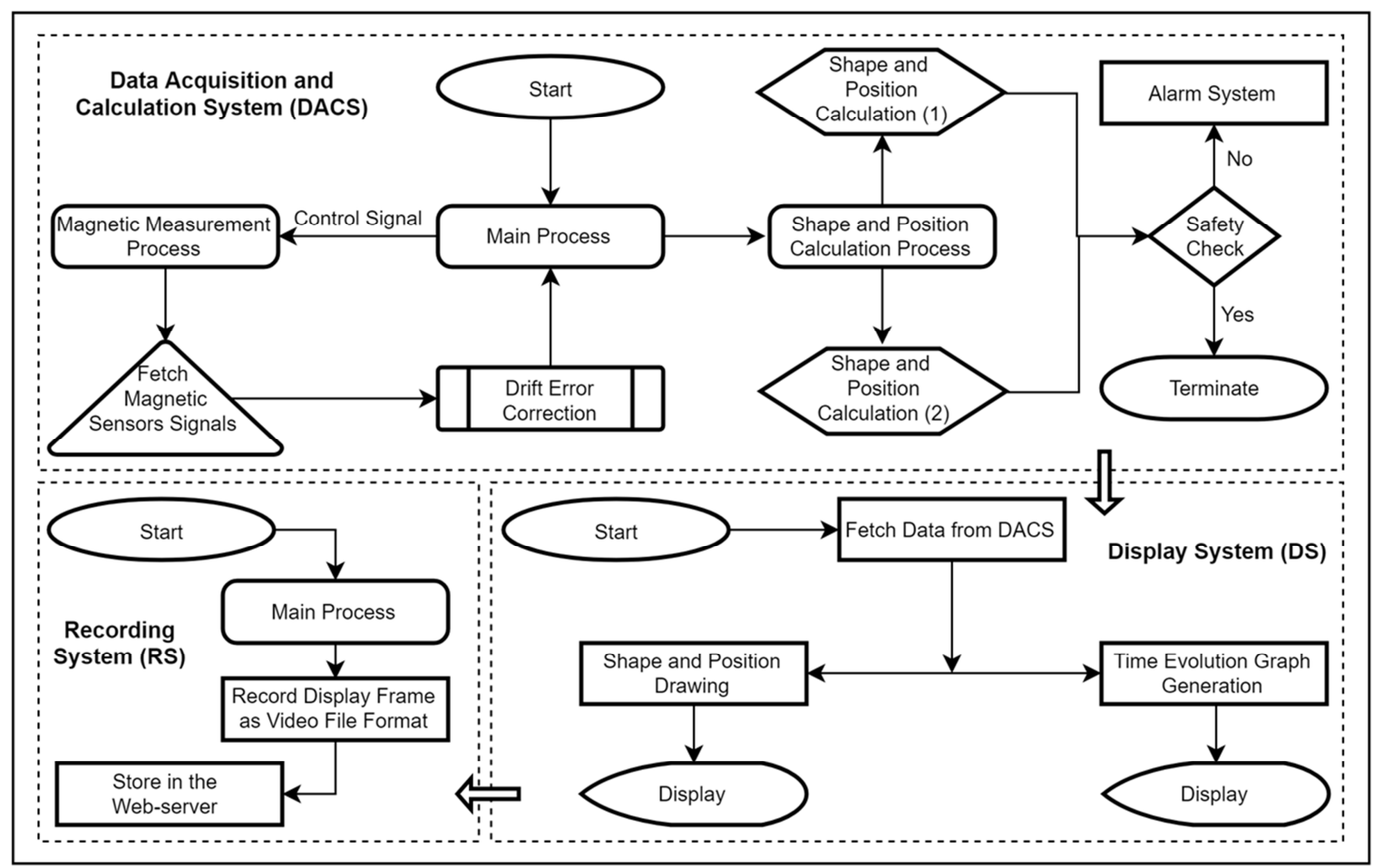

Figure 3. The control and data flow diagram of the real-time display system.

\section{Results}

We have designed and developed a real-time display system to monitor the plasma shape and position during the discharge of the tokamak with the video recording and the emergency safety notification sub-systems. According to the designing plan, the hardware assembling, configuration and integration for the sub-systems have been completed. The computational code and software have been developed and installed in the system according to the overall design; hardware configuration, data flow, data acquisition, error correction, data sharing and calculation mechanisms. The details of these processes have been discussed in this paper.

The system can display the actual shape of the last closed flux surface of the poloidal cross-section and its position on the major radius at a big screen placed inside the tokamak control room. In addition, the time evolution graphs of the plasma current, poloidal field coils currents, emission of $H_{\alpha}$, transport of the oxygen impurity, major radius, minor radius, aspect ratio, plasma elongation and triangularity are also displayed at that big screen in real-time. The video recording sub-system can record the entire display frame as well as the time evolution graphs as video files. The files are available in online just after the shot. The emergency safety notification sub-system can detect the occurrence of the abnormal situations and can generate safety alarm.

\section{Conclusion}

The real-time plasma shape and position display system is a practical system to monitor the plasma shape and position that are produced inside the vacuum vessel. This system is designed and developed with the real-time hardware and software. The standard structures of tokamak experimental facilities are considered during the design and development phases. The magnetic signals of the tokamak experiments are obtained as numerical integration and the drift errors are removed by using FPGA technology. To increase the functionality of the system two sub-systems (i) recording system and (ii) emergency safety notification system are introduced with it. Two different calculation methods are implemented in this system to ensure the precise and accurate 
calculations. As a result, the display system shows the actual shape of the last closed flux surface and its position on the major radius in real-time. The time evolution graphs of $I_{P}, I_{P F \mathrm{~s}}$, emission of $H_{\alpha}$, transport of the oxygen impurity, $R, a$, aspect ratio, $\kappa$ and $\delta$ are also precise and accurate. The collaborative researchers can participate with the experiment and can able to provide valuable comments during the experiments from the remote place by observing the recorded video files. The emergency safety notification system is ensured the safety and security of the overall experimental facilities and research environment.

\section{References}

[1] Anand, Himank, et al., "Plasma shape and position controller design for advance plasma configurations in TCV," APS Meeting Abstracts. Nov. 2015.

[2] S. Hara, K. Takeuchi, M. Abe and M. Otsuka, "Development of a real time visualization system for the shape of a tokamak plasma cross section," Review of scientific instruments, vol. 65 (11), pp. 3434-8, Nov. 1994.

[3] https://www.iter.org/newsline/128/247

[4] Zhang, X., Hu, et al., "The development of data acquisition and remote real-time display system for EAST NBI," Journal of Fusion Energy, Vol. 32 (5), pp. 566-569, 2013.

[5] Shu, Shuangbao, et al., "Plasma image edge detection based on the visible camera in the EAST device," SpringerPlus, vol. 5 (1), p. 2050, 2016.

[6] Ellis, J., et al., "A real time plasma boundary determination and display system using transputers," Proceedings of the 18th Symposium on Fusion Technology, pp. 743-746. 1994.
[7] Puig Sitjes, A., et al., "Wendelstein 7-x near real-time image diagnostic system for plasma-facing components protection," Fusion Science and Technology, vol. 74.1-2, pp. 116-124, 2018.

[8] Hasegawa, Makoto, et al., "Modification of plasma control system and hot-wall temperature control system for long-duration plasma sustainment in QUEST," Fusion Engineering and Design, vol. 129, pp. 202-206, 2018.

[9] Hartfuss, H. J., R. König, and A. Werner, "Diagnostics for steady state plasmas," Plasma physics and controlled fusion, vol. 48 (10), p. R83, 2006.

[10] Rodríguez, Alfonso, et al., "Fpga-based high-performance embedded systems for adaptive edge computing in cyber-physical systems: The artico3 framework," Sensors, vol. 18 (6), p. 1877, 8 June 2018.

[11] Lidozzi, Alessandro, et al., "Towards LabVIEW and system on module for power electronics and drives control applications," Proceedings of the IECON 2016-42nd Annual Conference of the IEEE Industrial Electronics Society. IEEE, 2016.

[12] Hasegawa, M., et al., "Development of plasma control system for divertor configuration on QUEST," Fusion Engineering and Design, vol. 88 (6-8), pp. 1074-1077, 2013.

[13] Kurihara, K., "A new shape reproduction method based on the Cauchy-condition surface for real-time tokamak reactor control," Fusion engineering and design, vol. 51, pp. 1049 (8), 2000 .

[14] Nakamura, Kazuo, et al. "Characteristics of SVD in ST Plasma Shape Reproduction Method Based on CCS," Plasma Fusion Res., vol. 8, P. 1048, 2009.

[15] K. Nakamura et al., "Shape reconstruction of RF-driven divertor plasma on QUEST," IEEE Trans. Plasma Sci. vol. 42 (9), p. 2309, 2014. 